\title{
Topological Conjugacy between Two Kinds of Nonlinear Differential Equations via Generalized Exponential Dichotomy
}

\author{
Xiaodan Chen and Yonghui Xia \\ Department of Mathematics, Zhejiang Normal University, Jinhua 321004, China \\ Correspondence should be addressed to Yonghui Xia, yhxia@zjnu.cn
}

Received 3 July 2011; Accepted 15 August 2011

Academic Editor: Yuri V. Rogovchenko

Copyright (C) 2011 X. Chen and Y. Xia. This is an open access article distributed under the Creative Commons Attribution License, which permits unrestricted use, distribution, and reproduction in any medium, provided the original work is properly cited.

Based on the notion of generalized exponential dichotomy, this paper considers the topological decoupling problem between two kinds of nonlinear differential equations. The topological equivalent function is given.

\section{Introduction and Motivation}

Well-known Hartman's linearization theorem for differential equations states that a 1:1 cor-respondence exists between solutions of a linear autonomous system $\dot{x}=A x$ and those of the perturbed system $\dot{x}=A x+f(x)$, as long as $f$ fulfills some goodness conditions, like smallness, continuity, or being Hartman [1]. Based on the exponential dichotomy, Palmer [2] extended this result to the nonautonomous system. Some other improvements of Palmer's linearization theorem are reported in the literature. For examples, one can refer to Shi [3], Jiang [4], and Reinfelds [5, 6]. Recently, Xia et al. [7] generalized Palmer's linearization theorem to the dynamic systems on time scales. Consider the linear system

$$
\dot{x}=A(t) x,
$$

where $x \in \mathbb{R}^{n}$ and $A(t)$ is a $n \times n$ matrix function. 
Definition 1.1. System (1.1) is said to possess an exponential dichotomy [8] if there exists a projection $P$ and constants $K>0, \alpha>0$ such that

$$
\begin{array}{r}
\left\|U(t) P U^{-1}(s)\right\| \leq K e^{-\alpha(t-s)}, \quad \text { for } s \leq t, s, t \in \mathbb{R}, \\
\left\|U(t)[I-P] U^{-1}(s)\right\| \leq K e^{\alpha(t-s)}, \quad \text { for } t \leq s, s, t \in \mathbb{R}
\end{array}
$$

hold, where $U(t)$ is a fundamental matrix of linear system $\dot{x}=A(t) x$.

However, Lin [9] argued that the notion of exponential dichotomy considerably restricts the dynamics. It is thus important to look for more general types of hyperbolic behavior. Lin [9] proposed the notion of generalized exponential dichotomy which is more general than the classical notion of exponential dichotomy.

Definition 1.2. System (1.1) is said to have a generalized exponential dichotomy if there exists a projection $P$ and $K \geq 0$ such that

$$
\begin{gathered}
\left|U(t) P U^{-1}(s)\right| \leq K \exp \left(-\int_{s}^{t} \alpha(\tau) d \tau\right), \quad(t \geq s), \\
\left|U(t)(I-P) U^{-1}(s)\right| \leq K \exp \left(\int_{s}^{t} \alpha(\tau) d \tau\right), \quad(t \leq s),
\end{gathered}
$$

where $\alpha(t)$ is a continuous function with $\alpha(t) \geq 0$, satisfying $\lim _{t \rightarrow+\infty} \int_{0}^{t} \alpha(\xi) d \xi=$ $+\infty, \lim _{t \rightarrow-\infty} \int_{t}^{0} \alpha(\xi) d \xi=+\infty$.

Example 1.3. Consider the system

$$
\left(\begin{array}{c}
\dot{x}_{1} \\
\dot{x}_{2}
\end{array}\right)=\left(\begin{array}{cc}
-\frac{1}{\sqrt[3]{|t|+1}} & 0 \\
0 & \frac{1}{\sqrt[3]{|t|+1}}
\end{array}\right)\left(\begin{array}{l}
x_{1} \\
x_{2}
\end{array}\right)
$$

Then, system (1.4) has a generalized exponential dichotomy, but the classical exponential dichotomy cannot be satisfied.

For this reason, basing on generalized exponential dichotomy, we consider the topological decoupling problem between two kinds of nonlinear differential equations. We prove that there is a $1: 1$ correspondence existing between solutions of topological decoupling systems, namely, $\dot{x}(t)=A(t) x(t)+f(t, x)$ and $\dot{x}(t)=A(t) x(t)+g(t, x)$. 
International Journal of Differential Equations

\section{Existence of Equivalent Function}

Consider the following two nonlinear nonautonomous systems:

$$
\begin{aligned}
& \dot{x}=A(t) x+f(t, x), \\
& \dot{x}=A(t) x+g(t, x),
\end{aligned}
$$

where $x \in \mathbb{R}^{n}, A(t), B(t)$ are $n \times n$ matrices.

Definition 2.1. Suppose that there exists a function $H: \mathbb{R} \times \mathbb{R}^{n} \rightarrow \mathbb{R}^{n}$ such that

(i) for each fixed $t, H(t, \cdot)$ is a homeomorphism of $\mathbb{R}^{n}$ into $\mathbb{R}^{n}$;

(ii) $H(t, x) \rightarrow \infty$ as $|x| \rightarrow \infty$, uniformly with respect to $t$;

(iii) assume that $G(t, \cdot)=H^{-1}(t, \cdot)$ has property (ii) too;

(iv) if $x(t)$ is a solution of system (2.1), then $H(t, x(t))$ is a solution of system (2.2).

If such a map $H$ exists, then (2.1) is topologically conjugated to (2.2). $H$ is called an equivalent function.

Theorem 2.2. Suppose that $\dot{x}=A(t) x$ has a generalized exponential dichotomy. If $f(t, x), g(t, x)$ fulfill

$$
\begin{gathered}
|f(t, x)| \leq F(t), \\
\left|f\left(t, x_{1}\right)-f\left(t, x_{2}\right)\right| \leq r(t)\left|x_{1}-x_{2}\right|, \\
|g(t, x)| \leq G(t), \\
\left|g\left(t, x_{1}\right)-g\left(t, x_{2}\right)\right| \leq r(t)\left|x_{1}-x_{2}\right|, \\
N(t, F, G) \leq B, \\
N(t, r) \leq L<1,
\end{gathered}
$$

where

$$
\begin{gathered}
N(t, F, G)=\int_{-\infty}^{t} K \exp \left(-\int_{s}^{t} \alpha(\varphi) d \varphi\right)(F(s)+G(s)) d s \\
+\int_{t}^{+\infty} K \exp \left(\int_{s}^{t} \alpha(\varphi) d \varphi\right)(F(s)+G(s)) d s \\
N(t, r)=\int_{-\infty}^{t} K \exp \left(-\int_{s}^{t} \alpha(\varphi) d \varphi\right) r(s) d s+\int_{t}^{+\infty} K \exp \left(\int_{s}^{t} \alpha(\varphi) d \varphi\right) r(s) d s,
\end{gathered}
$$

where $F(t), G(t), r(t) \geq 0$ are integrable functions and $B, L$ are positive constants, then the nonlinear 
nonautonomous system (2.1) is topologically equivalent to the nonlinear nonautonomous system (2.2). Moreover, the equivalent functions $H(t, x), G(t, y)$ fulfill

$$
|H(t, x)-x| \leq B, \quad|G(t, y)-y| \leq B
$$

In what follows, we always suppose that the conditions of Theorem 2.2 are satisfied. Denote that $X\left(t, t_{0}, x_{0}\right)$ is a solution of (2.2) satisfying the initial condition $X\left(t_{0}\right)=x_{0}$ and that $Y\left(t, t_{0}, y_{0}\right)$ is a solution of (2.1) satisfying the initial condition $Y\left(t_{0}\right)=y_{0}$. To prove the main results, we first prove some lemmas.

Lemma 2.3. For each $(\tau, \xi)$, system

$$
z^{\prime}=A(t) z-f(t, X(t, \tau, \xi))+g(t, X(t, \tau, \xi)+z)
$$

has a unique bounded solution $h(t,(\tau, \xi))$ with $|h(t,(\tau, \xi))| \leq B$.

Proof. Let $\mathbb{B}$ be the set of all the continuous bounded functions $x(t)$ with $|x(t)| \leq B$. For each $(\tau, \xi)$ and any $z(t) \in \mathbb{B}$, define the mapping $T$ as follows:

$$
\begin{aligned}
T z(t)= & \int_{-\infty}^{t} U(t) P U^{-1}(s)[g(s, X(s, \tau, \xi)+z)-f(s, X(s, \tau, \xi))] d s \\
& -\int_{t}^{+\infty} U(t)(I-P) U^{-1}(s)[g(s, X(s, \tau, \xi)+z)-f(s, X(s, \tau, \xi))] d s .
\end{aligned}
$$

Simple computation leads to

$$
\begin{aligned}
|T z(t)| \leq & \int_{-\infty}^{t}\left|U(t) P U^{-1}(s)\right|(F(s)+G(s)) d s \\
& +\int_{t}^{+\infty}\left|U(t)(I-P) U^{-1}(s)\right|(F(s)+G(s)) d s \\
\leq & \int_{-\infty}^{t} K \exp \left(-\int_{s}^{t} \alpha(\varphi) d \varphi\right)(F(s)+G(s)) d s \\
& +\int_{t}^{+\infty} K \exp \left(\int_{s}^{t} \alpha(\varphi) d \varphi\right)(F(s)+G(s)) d s \\
\leq & B,
\end{aligned}
$$


which implies that $T$ is a self-map of a sphere with radius $B$. For any $z_{1}(t), z_{2}(t) \in \mathbb{B}$,

$$
\begin{aligned}
\left|T z_{1}(t)-T z_{2}(t)\right| \leq & \int_{-\infty}^{t}\left|U(t) P U^{-1}(s)\right| r(s)\left(z_{1}(s)-z_{2}(s)\right) d s \\
& +\int_{t}^{+\infty}\left|U(t)(I-P) U^{-1}(s)\right| r(s)\left(z_{1}(s)-z_{2}(s)\right) d s \\
\leq & \left\|z_{1}-z_{2}\right\|\left[\int_{-\infty}^{t} K \exp \left(-\int_{s}^{t} \alpha(\varphi) d \varphi\right) r(s) d s\right. \\
& \left.+\int_{t}^{+\infty} K \exp \left(\int_{s}^{t} \alpha(\varphi) d \varphi\right) r(s) d s\right] \\
\leq & L\left\|z_{1}-z_{2}\right\| .
\end{aligned}
$$

Due to the fact that $L<1, T$ has a unique fixed point, namely, $z_{0}(t)$, and

$$
\begin{aligned}
z_{0}(t)= & \int_{-\infty}^{t} U(t) P U^{-1}(s)\left[g\left(s, X(s, \tau, \xi)+z_{0}(s)\right)-f(s, X(s, \tau, \xi))\right] d s \\
& -\int_{t}^{+\infty} U(t)(I-P) U^{-1}(s)\left[g\left(s, X(s, \tau, \xi)+z_{0}(s)\right)-f(s, X(s, \tau, \xi))\right] d s,
\end{aligned}
$$

it is easy to show that $z_{0}(t)$ is a bounded solution of (2.6). Now, we are going to show that the bounded solution is unique. For this purpose, we assume that there is another bounded solution $z_{1}(t)$ of (2.6). Thus, $z_{1}(t)$ can be written as follows:

$$
\begin{aligned}
z_{1}(t)= & U(t) U^{-1}(0) x_{0} \\
& +\int_{0}^{t} U(t) U^{-1}(s)\left[g\left(s, X(s, \tau, \xi)+z_{1}(s)\right)-f(s, X(s, \tau, \xi))\right] d s \\
= & U(t) U^{-1}(0) x_{0} \\
& +\int_{0}^{t} U(t)[P+(I-P)] U^{-1}(s)\left[g\left(s, X(s, \tau, \xi)+z_{1}(s)\right)-f(s, X(s, \tau, \xi))\right] d s \\
= & U(t) U^{-1}(0) x_{0} \\
& +\int_{-\infty}^{t} U(t) P U^{-1}(s)\left[g\left(s, X(s, \tau, \xi)+z_{1}(s)\right)-f(s, X(s, \tau, \xi))\right] d s \\
& -\int_{-\infty}^{0} U(t) P U^{-1}(s)\left[g\left(s, X(s, \tau, \xi)+z_{1}(s)\right)-f(s, X(s, \tau, \xi))\right] d s \\
& +\int_{0}^{+\infty} U(t)(I-P) U^{-1}(s)\left[g\left(s, X(s, \tau, \xi)+z_{1}(s)\right)-f(s, X(s, \tau, \xi))\right] d s \\
& -\int_{t}^{+\infty} U(t)(I-P) U^{-1}(s)\left[g\left(s, X(s, \tau, \xi)+z_{1}(s)\right)-f(s, X(s, \tau, \xi))\right] d s .
\end{aligned}
$$


Note that

$$
\begin{array}{rl}
\int_{-\infty}^{0} & U(t) P U^{-1}(s)\left[g\left(s, X(s, \tau, \xi)+z_{1}(s)\right)-f(s, X(s, \tau, \xi))\right] d s \\
& =U(t) U^{-1}(0) \int_{-\infty}^{0} U(0) P U^{-1}(s)\left[g\left(s, X(s, \tau, \xi)+z_{1}(s)\right)-f(s, X(s, \tau, \xi))\right] d s \\
& \leq U(t) U^{-1}(0)\left|\int_{-\infty}^{0} U(0) P U^{-1}(s)\left[g\left(s, X(s, \tau, \xi)+z_{1}(s)\right)-f(s, X(s, \tau, \xi))\right] d s\right| \\
& \leq U(t) U^{-1}(0) \int_{-\infty}^{0} K \exp \left(-\int_{s}^{0} \alpha(\varphi) d \varphi\right)(F(s)+G(s)) d s,
\end{array}
$$

which implies that $\int_{-\infty}^{0} U(0) P U^{-1}(s)\left[g\left(s, X(s, \tau, \xi)+z_{1}(s)\right)-f(s, X(s, \tau, \xi))\right] d s$ is convergent; denote it by $x_{1}$. That is,

$$
\int_{-\infty}^{0} U(t) P U^{-1}(s)\left[g\left(s, X(s, \tau, \xi)+z_{1}(s)\right)-f(s, X(s, \tau, \xi))\right] d s=U(t) U^{-1}(0) x_{1}
$$

Similarly,

$$
\int_{0}^{+\infty} U(t)(I-P) U^{-1}(s)\left[g\left(s, X(s, \tau, \xi)+z_{1}(s)\right)-f(s, X(s, \tau, \xi))\right] d s=U(t) U^{-1}(0) x_{2} .
$$

Therefore, it follows from the expression of $z_{1}(t)$ that

$$
\begin{aligned}
z_{1}(t)= & U(t) U^{-1}(0)\left(x_{0}-x_{1}+x_{2}\right) \\
& +\int_{-\infty}^{t} U(t) P U(s)\left[g\left(s, X(s, \tau, \xi)+z_{1}(s)\right)-f(s, X(s, \tau, \xi))\right] d s \\
& -\int_{t}^{+\infty} U(t)(I-P) U^{-1}(s)\left[g\left(s, X(s, \tau, \xi)+z_{1}(s)\right)-f(s, X(s, \tau, \xi))\right] d s .
\end{aligned}
$$

Noticing that $z_{1}(t)$ is bounded, $\int_{-\infty}^{t} U(t) P U(s)\left[g\left(s, X(s, \tau, \xi)+z_{1}(s)\right)-f(s, X(s\right.$, $\tau, \xi))] d s-\int_{t}^{+\infty} U(t)(I-P) U^{-1}(s)\left[g\left(s, X(s, \tau, \xi)+z_{1}(s)\right)-f(s, X(s, \tau, \xi))\right] d s$ is also bounded. So, $U(t) U^{-1}(0)\left(x_{0}-x_{1}+x_{2}\right)$ is bounded. But we see that $z^{\prime}=A(t) z$ does not have a nontrivial bounded solution. Thus, $U(t) U^{-1}(0)\left(x_{0}-x_{1}+x_{2}\right)=0$; it follows that

$$
\begin{aligned}
z_{1}(t)= & \int_{-\infty}^{t} U(t) P U^{-1}(s)\left[g\left(s, X(s, \tau, \xi)+z_{1}(s)\right)-f(s, X(s, \tau, \xi))\right] d s \\
& -\int_{t}^{+\infty} U(t)(I-P) U^{-1}(s)\left[g\left(s, X(s, \tau, \xi)+z_{1}(s)\right)-f(s, X(s, \tau, \xi))\right] d s .
\end{aligned}
$$


Simple calculating shows

$$
\begin{aligned}
\left|z_{1}(t)-z_{0}(t)\right| \leq & \int_{-\infty}^{t}\left|U(t) P U^{-1}(s)\right| r(s)\left|z_{1}(s)-z_{0}(s)\right| d s \\
& +\int_{t}^{+\infty}\left|U(t)(I-P) U^{-1}(s)\right| r(s)\left|z_{1}(s)-z_{0}(s)\right| d s \\
\leq & \left\|z_{1}-z_{0}\right\|\left[\int_{-\infty}^{t} K \exp \left(-\int_{s}^{t} \alpha(\varphi) d \varphi\right) r(s)\right. \\
& \left.+\int_{t}^{+\infty} K \exp \left(\int_{s}^{t} \alpha(\varphi) d \varphi\right) r(s)\right] \\
\leq & L\left\|z_{1}-z_{0}\right\| .
\end{aligned}
$$

Therefore, $\left\|z_{1}-z_{0}\right\| \leq L\left\|z_{1}-z_{0}\right\|$, consequently $z_{1}(t) \equiv z_{0}(t)$. This implies that the bounded solution of (2.6) is unique. We may call it $h(t(\tau, \xi))$. From the above proof, it is easy to see that $|h(t,(\tau, \xi))| \leq B$.

Lemma 2.4. For each $(\tau, \xi)$, the system

$$
z^{\prime}=A(t) z+f(t, X(t, \tau, \xi)+z)-g(t, X(t, \tau, \xi))
$$

has a unique bounded solution $\tilde{g}(t,(\tau, \xi))$ and $|\tilde{g}(t,(\tau, \xi))| \leq B$.

Proof. The proof is similar to that of Lemma 2.3.

Lemma 2.5. Let $x(t)$ be any solution of the system (2.1), then $z(t)=0$ is the unique bounded solution of system

$$
z^{\prime}=A(t) z+f(t, x(t)+z)-f(t, x(t))
$$

Proof. Obviously, $z \equiv 0$ is a bounded solution of system (2.19). We show that the bounded solution is unique. If not, then there is another bounded solution $z_{1}(t)$, which can be written as follows:

$$
z_{1}(t)=U(t) U^{-1}(0) z_{1}(0)+\int_{0}^{t} U(t) U^{-1}(s)\left[f\left(s, X(s, \tau, \xi)+z_{1}(s)\right)-f(s, X(s, \tau, \xi))\right] d s
$$


By Lemma 2.3, we can get

$$
\begin{aligned}
z_{1}(t)= & \int_{-\infty}^{t} U(t) P U^{-1}(s)\left[f\left(s, X(s, \tau, \xi)+z_{1}(s)\right)-f(s, X(s, \tau, \xi))\right] d s \\
& -\int_{t}^{+\infty} U(t)(I-P) U^{-1}(s)\left[f\left(s, X(s, \tau, \xi)+z_{1}(s)\right)-f(s, X(s, \tau, \xi))\right] d s .
\end{aligned}
$$

It follows that

$$
\begin{aligned}
\left|z_{1}(t)\right| \leq & \int_{-\infty}^{t}\left|U(t) P U^{-1}(s)\right| r(s)\left|z_{1}(s)\right| d s \\
& +\int_{t}^{+\infty}\left|U(t)(I-P) U^{-1}(s)\right| r(s)\left|z_{1}(s)\right| d s \\
\leq & \int_{-\infty}^{t} K \exp \left(-\int_{s}^{t} \alpha(\varphi) d \varphi\right) r(s)\left|z_{1}(s)\right| d s \\
& +\int_{t}^{+\infty} K \exp \left(\int_{s}^{t} \alpha(\varphi) d \varphi\right) r(s)\left|z_{1}(s)\right| d s \\
\leq & L\left|z_{1}(t)\right| .
\end{aligned}
$$

That is, $\left\|z_{1}\right\| \leq L\left|z_{1}\right|$. Consequently, $z_{1}(t) \equiv 0$. This completes the proof of Lemma 2.5.

Lemma 2.6. Let $y(t)$ be any solution of the system (2.2), then $z(t)=0$ is the unique bounded solution of system

$$
z^{\prime}=A(t) z+g(t, y(t)+z)-g(t, y(t))
$$

Proof. Obviously, $z \equiv 0$ is a bounded solution of system (2.23). We will show that the bounded solution is unique. If not, then there is another bounded solution $z_{1}(t)$. Then, $z_{1}(t)$ can be written as follows:

$$
z_{1}(t)=U(t) U^{-1}(0) z_{1}(0)+\int_{0}^{t} U(t) U^{-1}(s)\left[g\left(s, Y(s, \tau, \xi)+z_{1}(s)\right)-g(s, Y(s, \tau, \xi))\right] d s
$$

By Lemma 2.3, we can get

$$
\begin{aligned}
z_{1}(t)= & \int_{-\infty}^{t} U(t) P U^{-1}(s)\left[g\left(s, Y(s, \tau, \xi)+z_{1}(s)\right)-g(s, Y(s, \tau, \xi))\right] d s \\
& -\int_{t}^{+\infty} U(t)(I-P) U^{-1}(s)\left[g\left(s, Y(s, \tau, \xi)+z_{1}(s)\right)-g(s, Y(s, \tau, \xi))\right] d s .
\end{aligned}
$$


Then, it follows that

$$
\begin{aligned}
\left|z_{1}(t)\right| \leq & \int_{-\infty}^{t}\left|U(t) P U^{-1}(s)\right| r(s)\left|z_{1}(s)\right| d s \\
& +\int_{t}^{+\infty}\left|U(t)(I-P) U^{-1}(s)\right| r(s)\left|z_{1}(s)\right| d s \\
\leq & \int_{-\infty}^{t} K \exp \left(-\int_{s}^{t} \alpha(\varphi) d \varphi\right) r(s)\left|z_{1}(s)\right| d s \\
& +\int_{t}^{+\infty} K \exp \left(\int_{s}^{t} \alpha(\varphi) d \varphi\right) r(s)\left|z_{1}(s)\right| d s \\
\leq & L\left|z_{1}(t)\right| .
\end{aligned}
$$

That is, $\left\|z_{1}\right\| \leq L\left\|z_{1}\right\|$. Consequently, $z_{1}(t) \equiv 0$. This completes the proof of Lemma 2.6.

Now, we define two functions as follows:

$$
\begin{aligned}
& H(t, x)=x+h(t,(t, x)), \\
& G(t, x)=y+\tilde{g}(t,(t, y)) .
\end{aligned}
$$

Lemma 2.7. For any fixed $\left(t_{0}, x_{0}\right), H\left(t, X\left(t, t_{0}, x_{0}\right)\right)$ is a solution of the system (2.2).

Proof. Replace $(\tau, \xi)$ by $(t, X(t, \tau, \xi))$ in (2.6); system (2.6) is not changed. Due to the uniqueness of the bounded solution of (2.6), we can get that $h\left(t,\left(t, X\left(t, t_{0}, x_{0}\right)\right)\right)=h\left(t,\left(t_{0}, x_{0}\right)\right)$. Thus,

$$
H\left(t, X\left(t, t_{0}, x_{0}\right)\right)=X\left(t, t_{0}, x_{0}\right)+h\left(t,\left(t_{0}, x_{0}\right)\right) .
$$

Differentiating it, noticing that $X\left(t, t_{0}, x_{0}\right), h\left(t,\left(t_{0}, x_{0}\right)\right)$ are the solutions of the (2.1), and (2.6), respectively; therefore, we can obtain

$$
\begin{aligned}
{\left[H\left(t, X\left(t, t_{0}, x_{0}\right)\right)\right]^{\prime}=} & A(t) X\left(t, t_{0}, x_{0}\right)+f\left(t, X\left(t, t_{0}, x_{0}\right)\right)+A(t) h\left(t,\left(t_{0}, x_{0}\right)\right) \\
& -f\left(t, X\left(t, t_{0}, x_{0}\right)\right)+g\left(t, X\left(t, t_{0}, x_{0}\right)+h\left(t,\left(t_{0}, x_{0}\right)\right)\right) \\
= & A(t) H\left(t, X\left(t, t_{0}, x_{0}\right)\right)+g\left(t, H\left(t, t_{0}, x_{0}\right)\right) .
\end{aligned}
$$

It indicates that $H\left(t, X\left(t, t_{0}, x_{0}\right)\right)$ is the solution of the system (2.2).

Lemma 2.8. For any fixed $\left(t_{0}, y_{0}\right), G\left(t, Y\left(t, t_{0}, y_{0}\right)\right)$ is a solution of the system (2.1).

Proof. The proof is similar to Lemma 2.7.

Lemma 2.9. For any $t \in \mathbb{R}, y \in \mathbb{R}^{n}, H(t, G(t, y)) \equiv y$. 
Proof. Let $y(t)$ be any solution of the system (2.2). From Lemma 2.8, $G(t, y(t))$ is a solution of system (2.1). Then, by Lemma 2.7, we see that $H(t, G(t, y(t)))$ is a solution of (2.2), written as $y_{1}(t)$. Denote $J(t)=y_{1}(t)-y(t)$. Differentiating, we have

$$
\begin{aligned}
J^{\prime}(t) & =y_{1}^{\prime}(t)-y^{\prime}(t) \\
& =A(t) y_{1}(t)+g\left(t, y_{1}(t)\right)-A(t) y(t)-g(t, y(t)) \\
& =A(t) J(t)+g(t, y(t)+J(t))-g(t, y(t)),
\end{aligned}
$$

which implies that $J(t)$ is a solution of system (2.23). On the other hand, following the definition of $H$ and $G$ and Lemmas 2.3 and 2.4, we can obtain

$$
\begin{aligned}
|J(t)| & =|H(t, G(t, y(t)))-y(t)| \\
& \leq|H(t, G(t, y(t)))-G(t, y(t))|+|G(t, y(t))-y(t)| \\
& =|h(t,(t, G(t, y(t))))|+|\tilde{g}(t,(t, y))| \\
& \leq 2 B .
\end{aligned}
$$

This implies that $J(t)$ is a bounded solution of system (2.23). However, by Lemma 2.6, system (2.23) has only one zero solution. Hence, $J(t) \equiv 0$, consequently $y_{1}(t) \equiv y(t)$, that is, $H(t, G(t, y))=y(t)$. Since $y(t)$ is any solution of the system (2.2), Lemma 2.9 follows.

Lemma 2.10. For any $t \in \mathbb{R}, x \in \mathbb{R}^{n}, G(t, H(t, x)) \equiv x$.

Proof. The proof is similar to Lemma 2.10 .

Now, we are in a position to prove the main results.

Proof of Theorem 2.2. We are going to show that $H(t, \cdot)$ satisfies the four conditions of Definition 2.1.

\section{Proof of Condition (i)}

For any fixed $t$, it follows from Lemmas 2.9 and 2.10 that $H(t, \cdot)$ is homeomorphism and $G(t, \cdot)=H^{-1}(t, \cdot)$.

\section{Proof of Condition (ii)}

From (2.27) and Lemma 2.3, we derive $|H(t, x)-x|=|h(t,(t, x))| \leq B$. So, $|H(t, x)| \rightarrow \infty$ as $|x| \rightarrow \infty$, uniformly with respect to $t$.

\section{Proof of Condition (iii)}

From (2.23) and Lemma 2.4, we derive $|G(t, y)-y|=|\tilde{g}(t,(t, y))| \leq B$. So, $|G(t, y)| \rightarrow \infty$ as $|x| \rightarrow \infty$, uniformly with respect to $t$. 


\section{Proof of Condition (iv)}

Using Lemmas 2.7 and 2.8, we easily prove that Condition (iv) is true.

Hence, systems (2.1) and (2.2) are topologically conjugated. This completes the proof of Theorem 2.2.

\section{Acknowledgments}

The authors would like to express their gratitude to the editor and anonymous reviewers for their careful reading which improved the presentation of this paper. This work was supported by the National Natural Science Foundation of China under Grant (no. 10901140) and ZJNSFC under Grant (no. Y6100029).

\section{References}

[1] P. Hartman, "On the local linearization of differential equations," Proceedings of the American Mathematical Society, vol. 14, pp. 568-573, 1963.

[2] K. J. Palmer, "A generalization of Hartman's linearization theorem," Journal of Mathematical Analysis and Applications, vol. 41, pp. 753-758, 1973.

[3] J. Shi, "An improvement of Harman's linearization thoerem," Science in China A, vol. 32, pp. 458-470, 2002.

[4] L. P. Jiang, "A generalization of Palmer's linearization theorem," Mathematica Applicata, vol. 24, no. 1, pp. 150-157, 2011 (Chinese).

[5] A. Reinfeld, "A generalized Grobman-Hartman theorem," Latviǔskĭ Matematicheskiu Ezhegodnik, vol. 29, pp. 84-88, 1985.

[6] A. Reinfelds, "A reduction theorem for systems of differential equations with impulse effect in a Banach space," Journal of Mathematical Analysis and Applications, vol. 203, no. 1, pp. 187-210, 1996.

[7] Y. Xia, J. Cao, and M. Han, "A new analytical method for the linearization of dynamic equation on measure chains," Journal of Differential Equations, vol. 235, no. 2, pp. 527-543, 2007.

[8] W. A. Coppel, Dichotomies in Stability Theory, vol. 629 of Lecture Notes in Mathematics, Springer, Berlin, Germany, 1978.

[9] M. Lin, "Generalized exponential dichotomy," Journal of Fuzhou University, vol. 10, no. 4, pp. 21-30, 1982 (Chinese). 


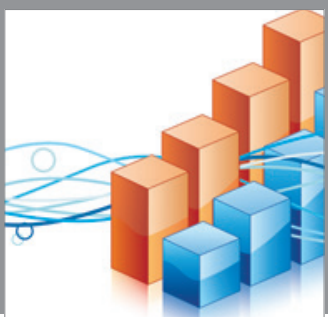

Advances in

Operations Research

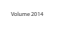

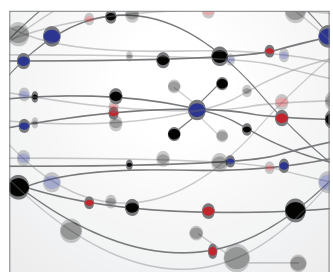

\section{The Scientific} World Journal
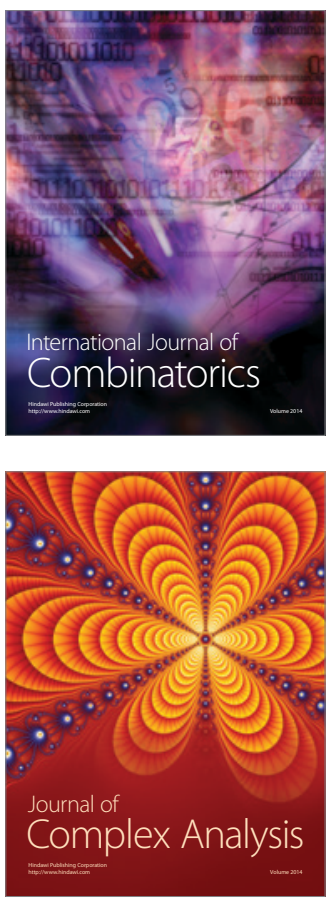

International Journal of

Mathematics and

Mathematical

Sciences
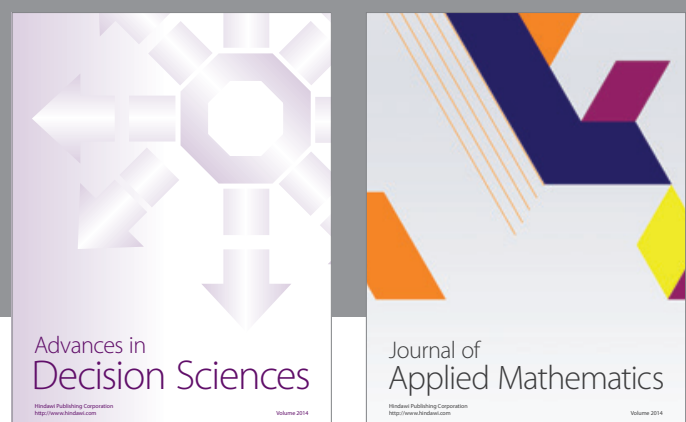

Journal of

Applied Mathematics
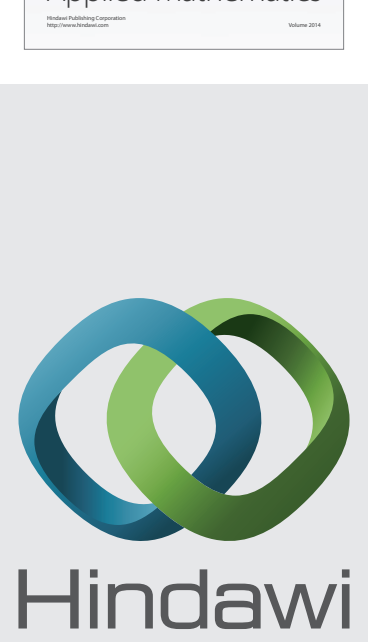

Submit your manuscripts at http://www.hindawi.com
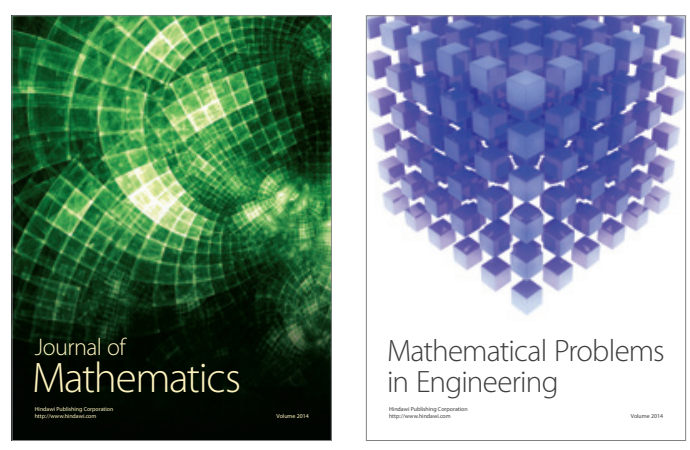

Mathematical Problems in Engineering
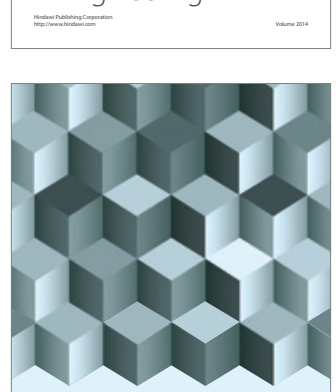

Journal of

Function Spaces
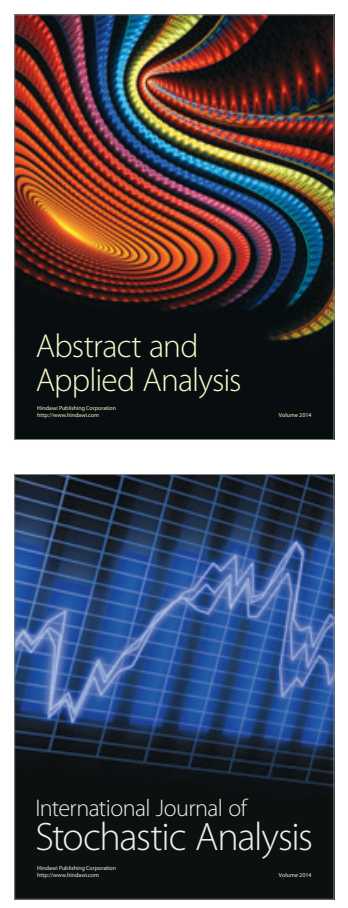

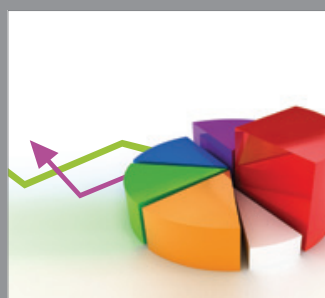

ournal of

Probability and Statistics

Promensencen
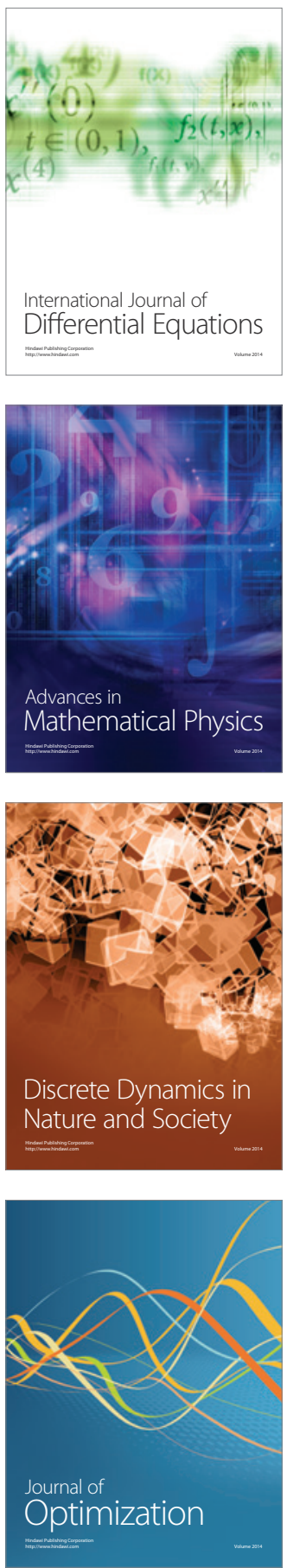